\section{Neuer Impfstoff gegen Tuberkulose}

Van Der Meeren O et al. Phase 2b Controlled Trial of M72/AS01E Vaccine to Prevent Tuberculosis. N Engl J Med 2018; 379: 1621 - 1621

Schätzungen zufolge ist ein Viertel der Weltbevölkerung mit Erregern der Tuberkulose (TB) infiziert, 5-10\% der Infizierten erkranken im Verlauf des Lebens an TB. Das erklärte Ziel der Weltgesundheitsorganisation WHO ist die Reduktion der TB-Inzidenz um $90 \%$ bis zum Jahr 2035. Aufgrund der zunehmenden Resistenzentwicklung ist dieses Ziel allein durch medikamentöse Therapie nicht zu erreichen.

\section{Hintergrund}

Tuberkulose-Impfstoffe zur Vermeidung der Infektion oder des Erkrankungsausbruchs werden dringend benötigt. Die bisherig zugelassenen Lebendimpfstoffe gegen Tuberkulose, attenuierte Mycobacterium bovis-Stämme vom Typ Bacille Calmette-Guérin, waren zwar gegen die Meningitis tuberculosa bei Kleinkindern erfolgreich, schützten aber nicht ausreichend gegen die pulmonale Tuberkulose und waren daher hinsichtlich der Elimination der TB nicht wirksam. Insbesondere bereits mit Mykobakterien infizierte Personen konnten durch bisherige Impfstoffe nicht geschützt werden. Neue Impfstoffe sind seit Langem in der Entwicklung.

Ein neuer Totimpfstoff zeigt nun in einer Phase 2b-Studie erste vielversprechende Ansätze. Der von der Firma GlaxoSmithKline entwickelte Impfstoff M72/AS01E besteht aus einem rekombinanten Fusionsprotein M72, das aus 2 immunogenen TB-Antigenen (Mtb32A und Mtb39A) entwickelt wurde, kombiniert mit dem AS01-Adjuvans, das bereits als Bestandteil des rekombinanten inzwischen in Europa zugelassenen ZosterTotimpfstoffs (Shingrix, GlaxoSmithKline) verwendet wird. Der Impfstoff wurde bereits in mehreren Phase 2-Studien untersucht und induziert eine gute humo- rale und T-Zell-vermittelte Immunität sowohl bei Menschen mit latenter als auch aktiver Tuberkulose, bei nicht infizierten Personen, bei BCG-geimpften Kindern und bei HIV-koinfizierten Patienten unter antiretroviraler Therapie.

\section{Studiendesign}

In 11 Zentren in Kenia, Südafrika und Sambia wurde der Impfstoff in einer randomisierten doppelblinden placebokontrollierten Studie an 3575 Probanden mit latenter M. tuberculosis-Infektion (LTBI) untersucht. Die Studienteilnehmer waren zwischen 18 und 50 Jahre alt, hatten keine wesentlichen Vorerkrankungen, insbesondere keine aktive Tuberkulose, und waren HIV-negativ. Die LTBI wurde durch einen positiven QuantiFERON-TB Gold In-Tube assay (QFT, Qiagen) nachgewiesen. Eine aktive Tuberkulose wurde durch Sputumuntersuchungen ausgeschlossen.

Die Probanden erhielten M72/AS01E oder Placebo in 2 Dosen im Abstand von einem Monat intramuskulär verabreicht. Die Nachverfolgung der Probanden hinsichtlich Entwicklung einer TB erfolgte bis 3 Jahre nach der Impfung.

\section{Ergebnisse}

1786 Probanden erhielten den Impfstoff M72/AS01E, 1787 erhielten Placebo. 1623 bzw. 1660 Teilnehmer konnten gemäß Protokoll ausgewertet werden. 10 der 1623 M72/AS01E-Geimpften und 22 der Nichtgeimpften entwickelten im 2,3 Jahre dauernden Verlaufsbeobachtungszeitraum eine mikrobiologisch bestätigte TB. Das entsprach einer Inzidenz von 0,3 versus 0,6 Fällen pro 100 Personenjahren, die Impfstoffwirksamkeit war damit $54 \%(p=0,04)$.

Der Wirkstoff war gut verträglich. Unerwünschte Wirkungen traten in der geimpften Gruppe mit 67,4\% signifikant häufiger als in der Placebogruppe mit $45,4 \%$ auf, insbesondere waren dies Schmerzen an der Impfstelle sowie grippeartige Symptome. Schwerwiegende unerwünschte Ereignisse und Todesfälle traten in beiden Gruppen gleich häufig auf (7 Todesfälle in der M72/AS01E-
Gruppe, davon 6 traumabedingt, versus 17 in der Placebogruppe, davon 8 traumabedingt), kein Todesfall wurde als studienbedingt bewertet. Bei den 33 in der Impfstoffgruppe entstandenen Schwangerschaften kam es nicht zu Missbildungen, 28 gesunde Kinder wurden geboren.

FAZIT

Diese Studie, die in einer großen Kohorte von bereits mit M. tuberculosis infizierten junger Menschen durchgeführt wurde, konnte zeigen, dass das Risiko einer TB durch einen Totimpfstoff signifikant um die Hälfte reduziert werden kann. Die Ergebnisse betonen die Notwendigkeit der weiteren Forschung an Impfstoffkandidaten und unterstützen die Hoffnung, dass effektive Impfstoffe gegen Tuberkulose entwickelt werden können.

\section{Dr. med. Hilte Geerdes-Fenge, Rostock}

